\title{
INFLUENCIA DE LAS REDES SOCIALES ACADÉMICAS EN LA CONSTRUCCIÓN DE LA IDENTIDAD DIGITAL LATINOAMERICANA
}

\author{
Wileidys Artigas* \\ Facultad de Ciencias Económicas y Sociales. Universidad del Zulia. \\ Ilya Casanova** \\ Facultad de Ciencias Médicas. Universidad Laica Eloy Alfaro de Manabí.
}

\begin{abstract}
Resumen: Para ofrecer una visión acerca de la relevancia que tiene en Latinoamérica la construcción de una identidad digital a través de las redes sociales académicas, se planteó como objetivo: examinar la presencia de investigadores latinoamericanos en ResearchGate, Academia y Autores Redalyc. La investigación fue empíricainductivista y se construyó un cuestionario a través de Google Forms para verificar la participación de investigadores latinoamericanos en las redes sociales académicas, se enviaron entre marzo y junio de 2019, 649 invitaciones vía correo electrónico y a través de las mismas redes; obteniendo 139 respuestas al respecto. Se concluyó que la presencia de los investigadores en las distintas redes no supera el 55\%, contrastando con otras investigaciones que resaltan la poca importancia y el subuso que se les ha dado a las herramientas que se ofrecen en dichas plataformas en la búsqueda del avance del conocimiento científico.

Palabras clave: redes sociales académicas; identidad digital latinoamericana; reputación científica; identidad digital del investigador; ResearchGate; Academia; Autores Redalyc.
\end{abstract}

Title: LATIN AMERICAN DIGITAL IDENTITY: INFLUENCE OF ACADEMIC SOCIAL NETWORKS.

Abstract: In order to offer a vision about the relevance of the construction of a digital identity through academic social networks in Latin America, the objective was: to examine the presence of Latin American researchers at ResearchGate, Academia and Autores Redalyc. The research was empirical-inductive and a questionnaire was constructed through Google Forms to verify the participation of Latin American researchers in the Academic Social Networks, 649 invitations were sent between March and June 2019 via email and through the same networks; getting 139 answers about it. It was concluded that the presence of researchers in the different networks does not exceed 55\%, contrasting with other investigations that highlight the little importance and the underuse that have been given to the tools offered in these platforms in the search for the advancement of the scientific knowledge.

Keywords: academic social networks; Latin American digital identity; scientific reputation; researcher digital identity; ResearchGate; Academia; Autores Redalyc.

Copyright: (C) 2020 Servicio de Publicaciones de la Universidad de Murcia (Spain). Este es un artículo de acceso abierto distribuido bajo los términos de la licencia Creative Commons Reconocimiento 4.0 Internacional (CC BY 4.0).

\section{INTRODUCCIÓN}

No es un tema nuevo hablar de la importancia de constituir redes y no sería nuevo tampoco relacionar la construcción de dichas redes, con temas de generación de conocimiento y difusión del mismo. En este sentido, ya se observaba que aquellos que se encontraban ligados a redes internacionales tenían privilegios y se volvían reconocidos como investigadores de prestigio en sus instituciones frente a aquellos que no logran o se resisten a integrarse. En aquel entonces, Kreimer (2006) hizo referencia a la masificación de las comunicaciones a través de medios electrónicos como principal elemento fortalecedor de dichas redes. Ya 15 años atrás, se vislumbraba que las comunicaciones iban a seguir avanzando para la mejora y desarrollo de la sociedad.

En esa época, Fernández (2008) señalaba que, aunque las publicaciones de impacto comenzaban a tomar importancia, no se podía dejar de lado, que más allá de hacerse notar, estaba la relevancia de la visión crítica del investigador. Destacaba también, el alcance no solo de hacer investigación, sino la presión por "hacer saber" lo que se investigaba.

Por su parte, Campos (2009, p. 2) resaltó que la generación de nuevos entornos y las plataformas de interrelación entre usuarios con mismas afinidades, establecían comunidades virtuales que se mantenían en un periodo de tiempo. Surgiendo, así, "la llamada Web 2.0 o red virtual convertida en espacio social de comunicación entre distintos usuarios

\footnotetext{
*wileartigas@gmail.com

** ilya.casanova@uleam.edu.ec
}

Recibido: 28-09-2019; 2ª versión: 29-03-2020; aceptado: 14-04-2020.

ARTIGAS, W. y CASANOVA, I. Influencia de las redes sociales académicas en la construcción de la identidad digital latinoamericana. Anales de Documentación, 2020, vol. 23, nº 2. Disponible en: http://dx.doi.org/10.6018/analesdoc.397551. 
e intervinientes, que aprovechan las herramientas informáticas de producción y gestión de la información para intercambiar contenidos”. Ya entonces, se hablaba de la creación de redes como ResearchGate - 2007- y el inicio del proceso de compartir directorios, buscadores, wikis, marcadores sociales y tags.

Considerando estas novedosas herramientas, Castaño (2009) ofrecía una visión del tema desde la educación, así se comenzaba a discutir los beneficios de la web 2.0, específicamente en cuanto al uso de podcast, e-learning, blogs y wikis. Es importante señalar que, con el avance de las aplicaciones web, se aprovecharon mejor estas herramientas en lo académico y por supuesto, esto toca el proceso de investigación. González-Díaz et al. (2015, p. 641) señalaban que "en su fase embrionaria, la aplicación de la Web 2.0 en la ciencia estuvo caracterizada por el uso de blogs. Sin embargo, son las redes sociales las que poseen un peso indiscutible en la web social”.

En el 2014 se hablaba de redes sociales generalistas, las cuales según Campos Freire:

“[...] son una nueva herramienta para la colaboración y la comunicación de la ciencia [...], aunque deberán perfeccionarse los sistemas de verificación, indexación, motores de búsqueda, tecnologías semánticas, reputación y evitar la brecha entre el conocimiento analógico y digital. Son nuevas herramientas y recursos tecnológicos que tanto los investigadores como las universidades deben tener presentes en sus estrategias de gestión. Y si el "índice h" de Google Scholar es un indicador de impacto y productividad, el índice de ResearchGate o RG Score -que mide a investigadores y universidades- es un indicador de impacto y popularidad. Su algoritmo registra el impacto de las publicaciones y la popularidad de las interacciones de los investigadores” (Campos Freire et al., 2014, p. 586).

Sobre el tema, Dorta-Contreras (2016), resalta que no solo a través de la colaboración se logra la visibilidad y citación de la producción científica y refiere la necesidad de incorporar además a los autores, las revistas e instituciones en las redes sociales. Esto hace referencia a las formas no convencionales de divulgar la ciencia y la creación de las métricas alternativas o altimetrías.

Arévalo, Cordón-García y Maltrás Barba (2016), refieren a altmetrics como la integración de todas las métricas, de manera que, da a conocer como los investigadores usan el internet para avanzar en sus procesos investigativos y señala igualmente a ResearchGate y Academia. Por otra parte, hacen referencia a la importancia de las mismas, pues inician desde el proceso de publicación, a diferencia de “[...] la Bibliometría ortodoxa que necesita que se publiquen otros trabajos y que referencien una investigación para poder obtener las primeras evidencias de su impacto” (p. 87).

Al respecto, Céspedes Villega et al. (2017), señalan que las fuentes de donde obtiene altmetrics los datos son variadas, ya que además de las convencionales agrega, las menciones en blogs, los "retwit” (se vuelve a publicar, otra persona respalda o coincide con lo expresado por el autor) o de cualquier medio electrónico en donde el material ha sido usado, lo cual da una visión más amplia de la visibilidad del documento, de allí la importancia que cobran la identidad digital en las redes sociales.

De cualquier forma, toda esta información recopilada, influye en la visibilidad y prestigio de los investigadores en el ámbito académico.

“El concepto de reputación científica conecta con el de identidad digital. Puede ser definida como el prestigio de un investigador obtenido gracias a la calidad e impacto de sus resultados de investigación. Las tecnologías de la información están condicionando este concepto, así, es posible hablar de reputación digital, online o ereputación, siendo su contrapartida la reputación offline. Necesariamente se plantea un estrecho vínculo entre ambos entornos de modo que, si el reconocimiento de un investigador se puede trasladar al contexto digital, una adecuada gestión de la identidad digital puede llevar a un mayor reconocimiento científico” (FernándezMarcial y González-Solar, 2015, p. 657).

Estos autores, definen la identidad digital como "el resultado del esfuerzo consciente que realiza el investigador por y para ser identificado y reconocido en un contexto digital” (Fernández-Marcial y González-Solar, 2015, p. 657). Definitivamente, la diferencia estará en aquellos que además de identificados pueden capitalizar sus interacciones y socializaciones en el ámbito académico e investigativo.

Los resultados del uso de la identidad digital podrán luego integrarse a los sistemas de evaluación de la ciencia, ya que se ha observado, que elementos como capacidad de integrarse en redes, cantidad de publicaciones no solo 
realizadas, sino leídas y compartidas denotan su influencia en el área de conocimiento donde se desempeña; influyendo no solo en su ámbito personal, sino también en las revistas e instituciones en las que participa. Como señalan Alperin y Rozemblum (2017, p. 238), quienes ven más allá del reporte de investigación, sobre todo hacen un llamado de atención a los encargados de los sistemas de aseguramiento de la calidad al puntualizar sobre:

"si la preocupación principal de la mayoría de los sistemas de evaluación de la ciencia está en la internacionalización, en difundir su ciencia, y en aumentar su impacto, no hay que olvidar que las revistas son [...] mucho más que un vehículo de comunicación de los resultados de la ciencia”.

Sin embargo, estos indicadores deben tomarse para lo que corresponda, ya que como señalan Waltman y Traag (2017), seria errado tomar el indicador de la revista para evaluar al investigador, ya que no necesariamente escribir en una revista muy citada determina la alta citación de un artículo en particular. Sobre este tema coinciden Tennant et al. (2019) quienes además añaden que la reputación de la revista no debe ser endosada a la del investigador que publica en ella. Esto pone en evidencia, el tema de los indicadores, que también requiere ser estudiado a detalle, pues tienen sus particularidades y no puede usarse de modo generalista. De cualquier forma, existe relación entre la propia identidad digital del investigador y la identidad digital de las revistas e instituciones en donde publica, pues en algún momento se entrelazan.

Lo que está claro, sin lugar a dudas es que la productividad y los indicadores han influido notablemente en las decisiones académicas e investigativas de las instituciones. Fernández-Marcial y González-Solar (2015, p. 657), señalan claramente que las vías de financiación han quedado condicionadas por la rentabilidad y productividad científicas, los indicadores de calidad se han convertido en factores clave para las universidades. Por otro lado, Orduña-Malea et al. (2016a, p. 306) expresan que, aunque mucho se ha hablado de lo negativo de estas redes y sus índices; uno de los beneficios de estas es el "[...] camino para la obtención de evidencias de impacto académico en determinadas áreas y disciplinas científicas”. Como señala Guédon (2019) el objetivo de estas plataformas no es solo la proyección, ya que con el acceso abierto esa posibilidad la tiene cualquiera, lo importante es el logro de prestigio para investigadores.

Al mismo tiempo, Orduña-Malea, Martín-Martín y Delgado López-Cózar (2016b) aclaran, que no se pueden dejar de lado las consecuencias de la proliferación de redes ya que la búsqueda constante de aumentar los indicadores ha generado un nuevo trastorno a los investigadores (que lo denomina dolor académico por un lado, e impactitis por el otro, relacionado con la obsesión por el factor de impacto), debido a la compulsión de acceso a las plataformas sociales académicas en cualquier momento y lugar, esperando nuevas descargas, citas o "me gusta”.

Sin embargo, aquí no finaliza todo, según Iglesias-García et al. (2015, p. 1136):

"Las redes sociales digitales científicas están todavía en fase de crecimiento y popularización, pero ya se han demostrado útiles como espacio colaborativo y de intercambio de conocimiento, por ello, no se deberían desaprovechar las posibilidades de circulación de la ciencia y de interactividad que ofrecen estas plataformas, ya que correrían el riesgo de convertirse en un repositorio más”.

Por las razones expuestas anteriormente, y en virtud de la importancia de las redes sociales académicas y la identidad digital para los investigadores, es importante el desarrollo de este artículo. El mismo, ofrece una visión acerca de la relevancia que tiene para Latinoamérica la construcción de una identidad digital a través de las redes sociales académicas (específicamente ResearchGate, Academia y Autores Redalyc). Tema de vital importancia, no solo para el investigador, sino también para las instituciones con miras a su evaluación. Así mismo, para las organizaciones orientadas al aseguramiento de la calidad, ya que no puede ignorarse el valor que han tomado estos elementos para el desarrollo de sistemas de evaluación acorde con los avances en la ciencia y la tecnología.

\section{REDES SOCIALES, REDES SOCIALES ACADÉMICAS Y ALTMETRICS}

Para hablar de redes sociales académicas, es preciso entender el tema de las redes sociales en su aspecto general. Campos Freire et al. (2014, p. 577) aclaran que:

"Las redes directas pueden ser horizontales o generalistas (Facebook, YouTube, Hi5, Sonico, MySpace, Tuenti) y verticales o especializadas por temática (profesional, identidad cultural, negocios, aficiones, viajes y otras temáticas), actividad (microblogging, juegos, geolocalización o georeferenciación, marcadores sociales 
y compartir objetos) y por contenido compartido (fotos, vídeos, documentos, presentaciones, noticias, lectura o ciencia). También se pueden clasificar por las características de sus relaciones: dirigidas (no bidireccionales) y no dirigidas (relaciones recíprocas e interactividad); explícitas (declaración de relación) e implícitas (deducidas del comportamiento)".

Dejando claro que estas redes han enfrentado un proceso de transformación, ya que del simple intercambio de datos de los años 90 se pasó a los sitios de redes digitales abiertas sobre un sistema que reconoce e interconecta perfiles (públicos o semipúblicos), amistades, comentarios, enlaces, búsquedas de conocimiento, citas, reputación, popularidad y contenidos de todo tipo (Campos Freire et al. 2014, p. 578). Por su parte, Alonso-Arroyo et al. (2016) señalan que las redes sociales han revolucionado la comunicación por su inmediatez, pasando de ser una herramienta a ser un fenómeno social.

Como señala Enríquez et al. (2015, p. 2) esta forma de hacer ciencia quizás se aparte de las practicas aceptadas por la comunidad científica, pero los investigadores no deben estar cerrados a estas nuevas tendencias, ya que:

"algunos de los textos difundidos de este modo han sido evaluados según alguna de las modalidades habituales en el mundo académico, mientras que otros carecen de esa evaluación, aunque se trata de formas de publicación que podrían en todos los casos ser reconocidas como científicas”.

Como resaltan Céspedes Villega et al. (2017, p. 114), la web 2.0 permitió a los usuarios interactuar y colaborar, generando comunidades virtuales, a diferencia de los sitios web estáticos donde solo se observaba contenido. Una de esas comunidades virtuales es la académica. En este sentido señalan que:

"Las redes sociales académicas, son un prototipo de la llamada web 2.0, que permiten a las personas o entidades relacionarse de forma rápida y paralela, así como compartir recursos de información y documentación de todo tipo. El uso de estas plataformas favorece los procesos de visibilidad de la ciencia, así como el establecimiento de relaciones profesionales. En las plataformas de la web 2.0 se generan una serie de indicadores que pueden utilizarse para la evaluación de la ciencia”.

Orduña-Malea et al. (2016b, p. 2) ya señalaban que "estas plataformas han comenzado a modelar la comunicación científica y bien podrían influir en la evaluación del impacto académico en el futuro”, si se dividen las métricas que pueden presentar estas redes en cinco categorías: bibliométricas (publicación y citación), uso, participación, valoración y conectividad social. En este sentido, el punto central está en las métricas o indicadores que desarrollan, ya que "en los últimos años ha habido avances en la búsqueda de este tipo de indicadores, en particular sobre las redes sociales en lo que hoy día se denomina altmetrics” (Alperin y Rozemblum, 2017, p. 238).

En este sentido, puede decirse que estas redes han innovado la forma a través de la cual se lleva a cabo la comunicación científica y obviamente han ampliado las posibilidades, logrando, así como señala Arévalo et al. (2016) tomar en consideración parámetros complementarios o alternativos. En este orden de ideas Arévalo et al. (2016, p. 83) señalan que:

"La influencia generada por los medios sociales ha sido calificada como Investigación 2.0, Social Reference, Altmetrics, (citando a Priem, Taraborelli y Groth 2010). Varios autores han investigado la influencia en Twitter, en Blogs y gestores de referencias. Sin lugar a dudas la mayor parte de los investigadores han trasladado sus actividades de investigación a la web y con el éxito de los medios sociales esta situación se ha hecho más evidente, ya que estas herramientas tienen más potencialidad para desarrollar un rango mayor de influencia académica que los entornos tradicionales de publicación (citando a Priem, Piwowar y Hemminger, 2011)”.

Altmetrics se alimenta de todos estos datos, no solo de las redes sociales académicas, sino también del resto de herramientas digitales disponibles en la web. Sin embargo, siendo las redes sociales académicas herramientas que dentro de sí contienen elementos de las métricas alternativas, es importante su revisión, ya que a través de ellas los investigadores pueden mostrar de cierta forma interacción con la comunidad en la cual se desarrolla.

Campos Freire et al. (2014) señalan como principales redes sociales académicas a ResearchGate, Academia y Mendeley; no obstante, para el caso de Latinoamérica se dejará de lado Mendeley y se incluirá la red de Autores Redalyc en función de que Mendeley no es usada comúnmente por los investigadores latinoamericanos. Por otro lado, 
también se ha observado un incremento en los perfiles de Google Académico, pero solo será usada como referencia en la cantidad, pues no ofrece la posibilidad de interacción y, por tanto, no puede clasificarse como red social académica.

\section{RESEARCHGATE, ACADEMIA Y AUTORES REDALYC}

De acuerdo con Alonso-Arroyo et al. (2016), la primera pregunta de los investigadores es ¿qué red social debo elegir? Y aunque sin duda alguna pudiera formar parte de varias, en ocasiones influyen muchos factores como el tiempo, líneas de investigación, colegas e instituciones adscritas.

Los investigadores entonces deciden la red a la cual adherirse y después viene el trabajo más amplio, hacerse activos en dicha red, que lleva una gran cantidad de tiempo y esfuerzo, ya que como señalan González-Díaz et al. (2015, p. 643) "no es suficiente con tener un número elevado de perfiles en las Redes Sociales Digitales Científicas (RSDC), sino que esos miembros tienen que ser activos: colgar gran número de trabajos, participar en los debates, etc.”.

ResearchGate ofrece un amplio repertorio de indicadores de rendimiento con el objetivo de medir la calidad y la cantidad de sus publicaciones. Destaca el indicador RG Score, calculado por un algoritmo propio, que integra indicadores bibliométricos y altimétricos mediante la medición de las publicaciones del investigador, las preguntas formuladas y contestadas por los iguales, las visitas y lecturas de los trabajos alojados en la plataforma, las descargas, las citas, entre otras ventajas (Alonso-Arroyo et al., 2016, p.139).

ResearchGate y Academia registran más de cien millones de documentos en línea y diez millones de visitas al mes. Laakso et al. (2017, p. 3) señalan que:

"In the last five years Academic Social Networks (ASNs) have become an option for researchers to further disseminate their research beyond primary publication in a scholarly journal, conference proceeding, or book. ASNs are social web platforms whose features are tailored specifically to the needs of researchers; however, the feature of particular relevance to this study is the functionality of making research publications available for anyone to download for free. As such we limit this study to ASNs that support that feature, e.g. ResearchGate and Academia.edu" ".

Por su parte, Enríquez et al. (2015, p. 5) resaltan que "ResearchGate es una red social y, como tal, comparte algunas características propias de estas”. Señala elementos interesantes como la validación de aptitudes y conocimientos, lo cual de cierta forma se constituye indirecta o directamente en un recurso de revisión por pares y la compara en este caso con LinkedIn. Orduña-Malea et al. (2016a, p. 305) dejan saber que:

"Como consecuencia tanto de su enorme popularidad como de los distintos indicadores que ofrece (no solo a nivel individual sino de instituciones de investigación y empresas), el interés de la comunidad científica por ResearchGate no ha tardado en manifestarse. A esta notoriedad contribuye sin lugar a dudas la amplia y compleja red de métricas disponibles”.

De hecho, los mismos autores concluyen su trabajo destacando que las prestaciones y servicios que ofrece ResearchGate permite enormes posibilidades a los investigadores, sin olvidar el amplio espacio a la mejora de los indicadores que aún se ponen en duda en algunos aspectos. De la experiencia personal de las autoras en la plataforma, se reconoce el valor agregado de tener no solo la posibilidad que los documentos subidos son indexados en Google Académico, sino que además ofrece la posibilidad de generar un DOIs a los documentos que no han sido compartidos o visualizados en otros medios de difusión, esto permite que estén siempre disponibles.

Céspedes Villega et al. (2017, p. 119) señalan que ResearchGate "posibilita que se compartan las publicaciones y se gane visibilidad, que se conecte y se colabore con otros investigadores, además se obtienen estadísticas y métricas sobre los perfiles y publicaciones y permite que se resuelvan cuestiones de forma colaborativa”. Por su parte, Enríquez et al. (2015, p. 5) precisan algunas otras bondades de ResearchGate:

“También permite la descarga de contenidos y, lo más interesante quizás, el poder compartirlos no solo dentro de ella, sino también por fuera del entorno cerrado de la red: en Facebook, Twitter, LinkedIn y Google+. A diferencia de las redes sociales antes mencionadas, ResearchGate proporciona para cada usuario un índice de visibilidad, el RG score, que podría pensarse como recurso parecido al índice de impacto de las publicaciones 
científicas tradicionales. Para un usuario dado, dicho índice se calcula teniendo en cuenta la naturaleza de las interacciones de otros investigadores con el material publicado por el usuario en cuestión, la participación en debates, la frecuencia de estas interacciones y el estatus de estos investigadores (medido mediante sus respectivos RG scores) que interactúan con el usuario o sus publicaciones. Además, ResearchGate también admite la creación de grupos”.

En el caso de Academia los usuarios pueden crear su propia página web para compartir los diferentes estudios y conferencias que suba a la plataforma, se puede enumerar sus intereses, a la vez que ofrece estadística de sus descargas y visitas a la página; otra de las ventajas es que permite saber qué palabras claves usan las personas para buscarlo en Google.

Romero-Rodríguez (2017) hace un análisis minucioso de las plataformas ResearchGate y Academia, para ello hace una comparación utilizando como parámetros: su impacto, perfiles, scores y métricas. Con referencia a la información aportada, el autor refiere que ResearchGate es más estratégico para el impacto efectivo (citas), aunque en Academia se consigan más lecturas, por la cantidad de usuarios registrados. Aun cuando la cantidad de usuarios de Academia puede verse impactante, menos de 1/3 son catalogados como usuarios activos (Romero-Rodríguez, 2016). Este hecho puede estar condicionado a que es relativamente sencillo registrarse y no es necesario un correo institucional, lo cual no da garantía que todos los usuarios sean investigadores activos, quizás por ello no cuentan con un número de documentos directamente proporcional a sus usuarios registrados. Sobre el tema Quintas (2016, p. 526) refiere que:

“Academia ostenta la posición más privilegiada en función de su posicionamiento, número de usuarios e impacto científico y académico mientras que ResearchGate dispone de un número mayor de documentos proporcionados por sus miembros. Será interesante analizar también las estrategias de ambas redes sociales de cara a su expansión y crecimiento”.

Por otro lado, Noorden (2014, p. 127) señala que luego de realizar una consulta a investigadores, esta arrojó que Academia "seemsless well-known tan ResearchGate: only 29\% of scientists in the survey were aware of it and just $5 \%$ visited regularly",.

De antemano se coincide con estas afirmaciones, sin embargo, este análisis no implica que no se utilicen ambas plataformas ya que como hasta ahora se ha señalado la identidad digital obliga a estar conectados en la mayor cantidad de redes disponibles y en las cuales puedas conectar e interactuar.

Ahora bien, Autores Redalyc es un recurso que brinda a los investigadores latinoamericanos múltiples ventajas dentro de las que se pueden mencionar: registro gratuito; no está ofreciendo constantemente que pagues por nuevos servicios; y permite conectar en tu perfil otras redes académicas e índices académicos como ORCID.org que además se encuentra enlazada con redes emergentes como Publons, la cual fue creada por Clarivate Analytics ${ }^{3}$. Según Hernández las bondades de la red Autores Redalyc estriban en que:

“coloca a disposición una hemeroteca en línea de acceso abierto, esto significa que permite leer, descargar y compartir artículos científicos a texto completo de forma gratuita, en apoyo a las labores académicas tanto de investigadores como de estudiantes. Además de generar indicadores que permiten conocer cuantitativa y cualitativamente la forma en la que se hace ciencia en Iberoamérica” (Hernández, 2015, p. 289).

En suma, y considerando los aspectos antes expuestos, se observa como las referidas redes (ResearchGate, Academia y Autores Redalyc) han ganado su espacio con respecto a los beneficios que ofrecen a los investigadores que se integran en ellas.

\section{METODOLOGÍA}

La investigación que se presenta es empírica-inductivista, y de acuerdo con la secuencia de desarrollo se cataloga como descriptiva, corresponde además con un estudio de tipo transeccional. Como herramienta para la recolección de los datos, se construyó un formulario, utilizando para ello los recursos de la plataforma de Google Forms con doble intención; en primera instancia verificar la participación de los investigadores latinoamericanos en este tipo de encuestas, y en segunda, recoger alguna información sobre las prácticas de investigación, aunque se tocaron diversos tópicos; uno de ellos estaba relacionado con pertenecer a una red académica o mejor conocida como red social 
académica (Academic Social Networks ASNs por Laakso et al. 2017), o RSDC (Campos-Freire, 2013 citado por González-Díaz et al. 2015).

Se enviaron 649 invitaciones para contribuir con el llenado del cuestionario, para esto se utilizaron bases de datos de varias vías: a) lista de correos de los doctorados en Ciencias Sociales, mención Gerencia, Ciencias Humanas, Ciencias Médicas, Ciencias de la Salud y Ciencias Odontológicas, los anteriores pertenecientes a la Universidad del Zulia (activos en el primer periodo de 2019); b) búsqueda en ResearchGate, por país, y envío aleatorio por instituciones de mayor tamaño de estos países, y c) envió por correo a investigadores conocidos por los autores (de un total 17 países), constatando que de estas invitaciones solo fueron respondidas el 28\% (139) en un promedio de 90 días. El proceso de envío se inició el 6 de marzo y la recepción concluyó el 30 de junio 2019, cuando se decide cerrar el formulario; el porcentaje bajo de respuesta, denota la falta de costumbre de los investigadores latinoamericanos a este tipo de solicitudes. A continuación, en la Tabla I se presenta la distribución por país de las encuestas recibidas:

\begin{tabular}{lc}
\hline Redes/países & Total por país \\
\hline Venezuela & 35 \\
Puerto Rico & 3 \\
Costa Rica & 2 \\
Colombia & 15 \\
Ecuador & 29 \\
México & 15 \\
Bolivia & 2 \\
El Salvador & 1 \\
Nicaragua & 3 \\
Perú & 9 \\
Chile & 5 \\
Paraguay & 4 \\
Argentina & 5 \\
Guatemala & 2 \\
Honduras & 1 \\
Cuba & 7 \\
Panamá & 1 \\
\hline TOTAL & 139 \\
\hline
\end{tabular}

Tabla I: Fuente: elaboración propia.

Para el análisis de los resultados se consultaron diversidad de artículos relacionados con el tema, para ello se utilizaron los motores de búsqueda académicos, utilizando palabras claves referidas a identidad digital y redes sociales académicas, de manera que se pudiese contrastar el estado del arte de la temática abordada.

\section{RESULTADOS}

A continuación, se presenta un cuadro resumen sobre la red a la cual pertenece cada uno de los encuestados, tomando en cuenta que solo existían 5 opciones (ResearchGate, Academia, Autores Redalyc, ninguna de las anteriores, otra: señale). Se debe aclarar que la idea del artículo no es analizar la veracidad de los índices que cada red genera, lo que se pretende es observar si el investigador latinoamericano la toma en cuenta como factor clave de proyección incluyéndose en la misma.

No se pretende ofrecer a través de este estudio la respuesta sobre los datos que influyen en la inclusión de investigadores en las redes sociales académicas, tampoco la importancia sobre el factor de impacto que estas redes desarrollan en su experiencia como investigador, lo que se pretende es tener una visión acerca del valor que tiene para investigadores latinoamericanos el pertenecer a alguna de estas redes en función de la relevancia que las mismas han tomado en los últimos años. 


\begin{tabular}{|c|c|c|c|c|c|}
\hline Redes/países & Total país & ResearchGate & Academia & Autores Redalyc & $\begin{array}{l}\text { En ninguna } \\
\text { red }\end{array}$ \\
\hline Venezuela & 35 & 18 & 19 & 10 & 9 \\
\hline Puerto Rico & 3 & 3 & 3 & 0 & 0 \\
\hline Costa Rica & 2 & 1 & 2 & 1 & 0 \\
\hline Colombia & 15 & 7 & 8 & 4 & 2 \\
\hline Ecuador & 29 & 12 & 13 & 7 & 9 \\
\hline México & 15 & 7 & 4 & 4 & 4 \\
\hline Bolivia & 2 & 0 & 0 & 0 & 2 \\
\hline El Salvador & 1 & 1 & 0 & 0 & 0 \\
\hline Nicaragua & 3 & 3 & 3 & 0 & 0 \\
\hline Perú & 9 & 2 & 2 & 3 & 5 \\
\hline Chile & 5 & 4 & 3 & 2 & 0 \\
\hline Paraguay & 4 & 3 & 0 & 0 & 1 \\
\hline Argentina & 5 & 4 & 1 & 0 & 1 \\
\hline Guatemala & 2 & 2 & 2 & 0 & 0 \\
\hline Honduras & 1 & 1 & 0 & 0 & 0 \\
\hline Cuba & 7 & 5 & 6 & 1 & 1 \\
\hline Panamá & 1 & 1 & 1 & 1 & 0 \\
\hline TOTAL & 139 & 74 & 67 & 33 & 34 \\
\hline$\%$ & 100 & $53,24 \%$ & $48,20 \%$ & $23,74 \%$ & $24,46 \%$ \\
\hline
\end{tabular}

Tabla II. Presencia de los investigadores latinoamericanos en redes sociales académicas. Fuente: elaboración propia a partir de los resultados de la investigación.

Cuando se les consultó a los investigadores sobre si pertenecían a una o más redes (Tabla II), del 100\% de los participantes, el 53,24\% pertenece a ResearchGate, el 48,20\% a Academia y el 23,74\% pertenece a Autores Redalyc. Se resalta que un $24,46 \%$ de autores señalaban no pertenecer a ninguna red.

Cabe destacar que al consultarles si participaban en otra red adicional a ResearchGate, Academia y Autores Redalyc, en la mayoría de los casos fue dejado en blanco. Para complementar esto, y tomando en cuenta la influencia Google Académico, en la misma encuesta se preguntó acerca de su uso, destacándose que el 91,4\% señaló que lo usaban como buscador de información y pocos lo referenciaron como un sitio donde colocar su perfil, lo cual hace de Google Académico un contador de citas y aglutinador de documentos más que una red social académica, al no permitir la interacción como el resto.

Noorden (2014) en un estudio similar donde consultó a un número importante de investigadores tanto sobre redes sociales en general (académicas y no académicas) como índices académicos, observó que la plataforma ResearchGate tiene mayor visibilidad entre los investigadores, solo superada por el índice Google Académico con un 90\%.

"The results confirm that ResearchGate is Giant certainly well-known (see 'Remarkable reach', and full results online at go.nature.com/jvx7pl). More than $88 \%$ of scientists and engineers said that they were aware of it slightly more than had heard of Google+ and Twitter with little difference between countries. Just under half said that they visit regularly, putting the site second only to Google Scholar, and ahead of Facebook and LinkedIn. Almost 29\% of regular visitors had signed up for a profile on ResearchGate in the past year ${ }^{4}$ (Noorden, 2014, p. 126-127).

Sobre este tema en Latinoamérica, Campos Freire et al. (2014) destacan que en los países andinos ResearchGate tiene más aceptación en las áreas de ciencias naturales y medicina, pero Academia, es más visible en las ramas sociohumanísticas. Por otra parte, refieren los autores, (2014, p. 582) que "La penetración de ResearchGate en Colombia alcanza al 50\% de su planta de profesorado, en Perú al 10\%, en Ecuador a otro 10\% y en Bolivia no pasa del 5\%. A diferencia de Colombia, pues, la penetración es baja aún”.

Ahora bien, aun cuando falta profundizar sobre el uso habitual de las redes en los investigadores latinoamericanos, se observa que algunas redes académicas son utilizadas de forma diferente por los académicos de distintos países, de allí por ejemplo que los resultados de este estudio coincidan con Calva Cabrera y Punín Larrea (2016), quienes señalan que los ecuatorianos priorizan a Academia sobre ResearchGate. Sin embargo, para estos autores el uso global de las redes por parte de los investigadores sigue siendo baja. 
Si se analizan los resultados obtenidos en el presente estudio, dentro del grupo consultado llama la atención Bolivia. Del 100\% de los que respondieron la encuesta, ninguno refirió pertenecer a alguna red social académica; por supuesto, aun cuando estos datos no son concluyentes, ya que sería necesario ampliar la muestra, de alguna manera da soporte a lo descrito por Campos Freire et al. (2014) quienes señalan la necesidad de revisar la importancia que le da el mundo académico de ese país, a estos temas.

No obstante, hay que tomar en cuenta que la cantidad de investigadores incluidos en estas redes y que no las usan habitualmente está sujeto a una serie de factores. Tal como señalan Fernández-Marcial y González-Solar (2015, p. 662):

“Los investigadores están siendo impulsados a crear perfiles por agentes externos, editoriales, agencias de evaluación, revistas científicas... Ello produce una cierta desviación de la naturaleza de estos sistemas que requieren un papel activo y sistemático del investigador. Puede darse el extremo de que un investigador para publicar una única vez en una revista cree uno o más perfiles que luego queden desactualizados”.

Otro de los temas que afecta la presencia de los investigadores en las redes sociales tiene que ver con lo señalado por Aguillo Caño et al. (2017), quienes realizaron un trabajo en Colombia comparando los perfiles existentes de ResearchGate (específicamente los indicadores) con los de COLCIENCIAS, observándose discrepancias en los datos, lo cual lleva a hacerse muchas preguntas sobre el desarrollo de los indicadores y la medida en que las altmetrics pudiesen contribuir a una evaluación académica más global. De los resultados arrojados en ese estudio se puede inferir las razones por la cual menos de la mitad de los investigadores colombianos se encuentran en ResearchGate, ya que, su sistema de evaluación difiere mucho con los elementos que aquí se expresan y, por tanto, prefieren obtener la evaluación formal de COLCIENCIAS y no dedicar tiempo a visibilizarse en las redes académicas.

Ahora bien, Robinson-García, Repiso y Torres-Salinas (2018, p. 464) puntualizan sobre este aspecto y señalan que: "El problema global no es tanto las fuentes, sino la estrechez de miras para incorporar nuevas visiones del impacto científico y nuevos formatos científicos, ignorando que el proceso científico va más allá de las citas”. Por su parte, Babini (2019, p. 2) deja claro que:

"Si queremos que en los procesos de evaluación en América Latina se valore también la producción publicada en acceso abierto en revistas de la región y al mismo tiempo ser inclusivos para abarcar una diversidad de publicaciones $-y$ no solo aquellas que son aceptadas por los servicios de indizaciones internacionales o regionales-, debemos trabajar juntos en el desarrollo de indicadores complementarios que muestren qué circulación, uso e impacto tienen los objetos digitales que publicamos en diversos formatos y en plataformas institucionales, nacionales y regionales”.

La realidad parece indicar una mayor coincidencia entre los investigadores, acerca de que aun cuando los score o indicadores de ResearchGate o Academia pueden servir de referencia para la construcción de nuevos indicadores de evaluación menos estáticos, hasta el momento la poca transparencia de las métricas utilizadas por estas redes sociales académicas los aleja de ser referente para el aseguramiento de la calidad de instituciones educativas. Sobre este aspecto, por ejemplo, Orduña-Malea et al. (2016a) señalan que el RG Score de ResearchGate es un indicador insuficiente, ya que la manera como se calcula y se asigna el peso de cada uno de estos indicadores es una caja negra. Pese a existir un razonable secreto comercial (ResearchGate es una empresa), la elaboración de indicadores bibliométricos que podrían ser usados en actividades de evaluación debería ser completamente transparente.

Por otro lado, se debe reconocer que no es Latinoamérica únicamente donde existe poca presencia de los investigadores en estas redes académicas. González-Díaz et al. (2015), determinaron que la presencia de profesores de universidades españolas en ResearchGate era insuficiente. Sin embargo, Dorta-Contreras (2016) añade un punto central, la factibilidad de las bases de datos gratuitas, puede ser uno de los elementos centrales por los cuales han ganado popularidad en los últimos años redes sociales académicas, sobre todo en el caso latinoamericano, ya que en países como Venezuela, las universidades no han podido pagar sus subscripciones a bases de datos y una de las formas que tienen los investigadores de dar a conocer su trabajo es solo a través de estas redes sociales académicas.

En este sentido, uno de los elementos más importantes a tomar en cuenta para el impulso de ResearchGate por ejemplo, tiene que ver según Orduña-Malea et al. (2016a, p. 309) con: 
"[...] servicios y datos de inestimable valor para los investigadores: permite depositar y tipificar una amplia diversidad de materiales académicos (incluso registrar de forma gratuita DOIs para estos), conocer el interés del sector profesional y docente en ciertos materiales (presentaciones, cursos, etc.), conocer las personas $e$ instituciones interesadas en tu trabajo, obtener datos de citas cada vez más completos según crece la cobertura de la plataforma (nada desdeñable en ciertas disciplinas como biomedicina), y fomentar la colaboración y networking académico".

No obstante, es un proceso en evolución, con el movimiento del Acceso Abierto, se siguen creando alianzas y van surgiendo nuevas redes sociales académicas que involucran instituciones, revistas y científicos. Tal es el caso de AmeliCA, la cual según Valenzuela (2019) se creó con la intención de contribuir a la integración en torno al diálogo de la comunicación científica en América Latina. En ella, participan la Organización de las Naciones Unidas para la Educación, la Ciencia y la Cultura; el Consejo Latinoamericano de Ciencias Sociales; la Red de Revistas Científicas de América Latina y el Caribe, España y Portugal; la Universidad Autónoma del Estado de México; la Universidad de Antioquia y la Universidad Nacional de La Plata. Por su parte, Microsoft está apostando por una red académica (Microsoft Academic), construyendo este artículo se recibió el correo de invitación para formar parte de la misma, lo cual muestra como siguen ampliándose las opciones.

En esta sintonía está también la Unión Europea quienes están enfocados en lograr que los artículos científicos sean de acceso libre antes del año 2020. Esta decisión se basa en tres principios fundamentales: 1 compartir el conocimiento libremente, 2 conseguir un acceso abierto y 3 lograr una reutilización de los datos de investigación. Mientras esto sucede, Sánchez-Juárez (2018, p. 57) recomienda a los autores que cuando tengan su artículo publicado y cuenten “con los derechos para su difusión, lo que sigue es comenzar una larga carrera de registros”, haciendo referencia al vaciado de información en redes e índices académicos para dar visibilidad a sus investigaciones y así lograr que dicho trabajo sea leído y citado.

Hablar de identidad digital es hablar de redes sociales académicas, ya lo señalaba Fernández-Marcial y GonzálezSolar (2015) cuando planteaban la visibilidad de la investigación y la identidad digital como una nueva dimensión a tomar en cuenta, cuando se hable de calidad de la investigación. Sin embargo, se debe poner atención a lo señalado por Sugimoto et al. (2016, p. 24).

"In this context, stakeholders that supply and demand altmetrics and the use of social media in academia must be cautious not to take the shadow for the substance, where measurable traces of research activities and impact become more important than the activities themselves ${ }^{5}$ ".

En este sentido, un grupo de académicos de alta trayectoria, ha tomado en sus manos ciertas decisiones para favorecer la generación de conocimiento y ralentizar la velocidad de publicación, generada desde las propias organizaciones casadas con editoriales que proyectan la evaluación a través de métricas rígidas, y donde se privilegia un tipo de indexación. Un ejemplo de esto es El Manifiesto de la Ciencia Lenta ${ }^{6}$ quienes lo suscriben señalan:

"Science needs time to read, and time to fail. Science does not always know what it might be at right now. Science develops unsteadily, with jerky moves and unpredictable leaps forward-at the same time, however, it creeps about on a very slow time scale, for which there must be room and to which justice must be done ${ }^{7}$ ".

Por otro lado, algunos investigadores están apoyando el surgimiento de revistas, que dentro de las instrucciones señalan que sus manuscritos se publicarán bajo una licencia Creative Commons Attribution (CC BY) ${ }^{8}$. Otros, se han venido alejando de las revistas científicas, sobre todo de aquellas que es necesario pagar por ver artículos propios, y han puesto su mirada en estas redes científicas y blog personales, logrando una identidad digital que en muchas ocasiones alcanza tanto o mayor número de citaciones de sus trabajos que si estuviesen en las revistas de alto impacto.

Por supuesto, sobre este tema se han tenido diversas posturas en los círculos académicos, ya que algunas políticas de evaluación académica, obligan a publicar en las revistas llamadas de alto impacto, no obstante, es momento de hacer un alto y poner atención en lo que se está transformando la investigación científica y sobre todo su socialización, ya que como refiere Bunge (2001, p. 30) “el conocimiento científico es comunicable: no es inefable sino expresable, no es privado sino público”, por tanto, no puede ser un negocio que se aleja en algunos casos de las buenas prácticas en investigación y que pone en riesgo el avance de la ciencia. 
También se ve con preocupación, que al privilegiarse solo a las revistas llamadas de alto impacto y dar la espalda a las institucionales noveles, se les está condenando a una muerte prematura, no por falta de calidad, sino que su permanencia e indexación requieren una serie de condiciones que involucra entre otras cosas el respaldo de sus propios investigadores, pero sobre todo de sus organizaciones. Esta práctica es habitual, por cuanto se prioriza en la evaluación académica estos indicadores y como ya se ha hecho referencia, es necesario que los mismos sean revisados, ¿qué tan cerca estamos de lograrlo? Al respecto, Robinson-García et al. (2018, p. 463) refieren que:

"En esta nueva era evaluativa, sin embargo, tanto las altimétricas como las nuevas bases de datos (con la excepción de Scopus) están aún lejos de cumplir con sus promesas y crear una alternativa viable al empleo de los indicadores bibliométricos tradicionales y al uso de la Web of Science como fuente principal”.

Hay que señalar que los actuales indicadores de evaluación, en muchos casos crean la necesidad de mantener el pago de bases de datos de consorcios editoriales que significan una erogación de dinero importante para las instituciones educativas, mayor incluso que el que reciben algunos investigadores para sus proyectos. En este sentido Arévalo et al. (2016, p. 86) señalan que altmetrics:

"podría en un futuro tener relación con la evaluación del profesorado y proceso de acreditación, proporcionando a los comités de revisión información complementaria sobre la investigación a efectos sociales o interdisciplinares, y también podría ser considerado como herramienta para la concesión y dotación de financiación de proyectos de investigación”.

Es innegable, que esto amerita volver a lo básico, a sentarse y unificar criterios desde la perspectiva, no de quienes administran o de quienes no investigan sino desde la mirada de investigadores.

\section{CONCLUSIONES}

Los investigadores no pueden negar que una nueva era ha llegado, esa era, es la de la identidad digital, y quieran o no, insertarse en ella terminará por hacerse necesario si se quiere avanzar en el reconocimiento del trabajo realizado. Hace algunos años para ser investigador bastaba con hacer unos cuantos proyectos con pares nacionales o muy a lo interno de las instituciones donde estaban adscritos y publicar algunos artículos o presentarse en una que otra conferencia o congreso internacional. Ahora no es suficiente, para poder ser investigador "reconocido" debe integrarse al panorama internacional, buscar el trabajo colaborativo con personas de otros países y publicar no solo en su idioma natal, sino en inglés y además en revista de impacto internacional. Para luego mostrar su avance a través de las redes sociales académicas en donde se encuentre, lo cual ayudará con el paso del tiempo a la construcción de su identidad digital. En suma, un investigador que no posea alguna medición de las métricas tradicionales sumadas a las métricas alternativas no será tomado en cuenta por la ciencia.

Si se asumen las altmetrics transparentes, como un mecanismo de evaluación más integral, se estaría resaltando, la importancia de que el investigador tenga capacidad de reconocer estos medios como parte de sus herramientas para hacerse visible y reformar su identidad digital. Anglada y Abadal (2018, p. 294) ya decían que “esta nueva manera de hacer ciencia — este nuevo paradigma - se fundamentaría en tres criterios: la ciencia debe ser abierta, colaborativa y hecha con y para la sociedad”.

Indiscutiblemente, no se puede negar la evolución, ni conformarse solo con reportar sus investigaciones, ahora debe aceptarse que también tendrá que contabilizarse el tiempo que pasan en las redes sociales académicas actualizando la información e interactuando con sus pares, las organizaciones tendrán que ceder y asignar horas como parte de su carga académica, ya que la visibilidad en redes académicas se puede afirmar que es parte de su accionar como investigador, contar con una identidad digital se hace cada vez más indispensable. Negarse a este movimiento puede condenar al investigador al ostracismo académico, la verdad toma su tiempo, pero la era de la identidad digital ha llegado para quedarse.

\section{NOTAS}

${ }^{1}$ En los últimos cinco años, las Redes Sociales Académicas (ASN, por sus siglas en inglés) se han convertido en una opción para que los investigadores difundan más su investigación más allá de la publicación primaria en una revista académica, conferencia o libro. Las ASN son plataformas web sociales cuyas características se adaptan específicamente a las necesidades de los investigadores; sin embargo, la característica de particular relevancia para este estudio es la funcionalidad de hacer que las publicaciones de investigación estén disponibles para que cualquiera las 
descargue de forma gratuita. Como tal, limitamos este estudio a las ASN que admiten esa función, ResearchGate y Academia. (TRADUCCIÓN PROPIA)

2 Academia parece poco conocida como ResearchGate: solo el 29\% de los científicos de la encuesta lo sabían y solo el 5\% visitó regularmente. (TRADUCCIÓN PROPIA)

$3<$ https://publons.com/about/home/>.

${ }^{4}$ Los resultados confirman que ResearchGate es un gigante ciertamente conocido (consulte "Alcance notable" y los resultados completos en línea en: go.nature.com/jvx7pl). Más del 88\% de los científicos e ingenieros dijeron que lo sabían, un poco más de lo que habían oído hablar de Google+ y Twitter, con poca diferencia entre países. Poco menos de la mitad dijo que lo visitan regularmente, colocando el sitio solo por detrás de Google Académico y por delante de Facebook y LinkedIn. Casi el 29\% de los visitantes regulares se habían inscrito en un perfil en ResearchGate en el último año.

${ }^{5}$ En este contexto, las partes interesadas que ofrecen y demandan métricas alternativas y el uso de las redes sociales en la academia deben ser cautelosos para no tomar la sombra de la sustancia, donde las huellas medibles de las actividades de investigación y el impacto se vuelven más importantes que las actividades mismas.

$6<$ http://slow-science.org/>.

${ }^{7}$ La ciencia necesita tiempo para leer y tiempo para fallar. La ciencia no siempre sabe lo que podría ser en este momento. La ciencia se desarrolla de forma inestable, con movimientos bruscos y saltos impredecibles hacia adelante; al mismo tiempo, sin embargo, avanza lentamente en una escala de tiempo muy lenta, para lo cual debe haber espacio y para lo que debe hacerse justicia. (TRADUCCIÓN PROPIA)

8 Tal es el caso de la revista Quantitative Science Studies (QSS), quienes apoyan primordialmente el acceso abierto bajo principios justos $<$ http://issi-society.org/blog/posts/2019/january/the-international-society-for-scientometrics-and-informetrics-ends-support-for-journal-ofinformetrics-launches-new-open-access-journal-quantitative-science-studies/>.

\section{BIBLIOGRAFÍA}

ALONSO-ARROYO, A.; ONTALBA-RUIPÉREZ, J.A.; GONZÁLEZ DE DIOS, J. y ALEIXANDRE-BENAVEN, R. Comunicación científica (XXXIII). El papel de las redes sociales en la difusión de la pediatría. Acta Pediátrica Española, 2016, vol. 74, n 5, p. 133-140.

AGUILLO CAÑO, I.F.; URIBE TIRADO, A. y LÓPEZ LÓPEZ, W. Visibilidad de los investigadores colombianos según sus indicadores en Google Scholar y ResearchGate. Diferencias y similitudes con la clasificación oficial del sistema nacional de ciencia-COLCIENCIAS. Revista Interamericana de Bibliotecología, 2017, vol. 40, nº 3, p. 221-230. Disponible en: <http://hdl.handle.net/10495/9400> [Consulta: 30 de junio de 2019]

ALPERIN, J.P. y ROZEMBLUM, C. La reinterpretación de visibilidad y calidad en las nuevas políticas de evaluación de revistas científicas. Revista Interamericana de Bibliotecología, 2017, vol. 40, nº 3, p. 231-241.

ANGLADA, L. y ABADAL, E. ¿Qué es la ciencia abierta? Anuario ThinkEPI, 2018, vol. 12, p. 292-298. Disponible en: https://doi.org/10.3145/thinkepi.2018.43.

ARÉVALO, J.A.; CORDÓN-GARCIA, J.A. y MALTRÁS BARBA, B. Altmetrics: medición de la influencia de los medios en el impacto social de la investigación. Cuadernos de Documentación Multimedia, 2016, vol. 27, nº 1, p. 75-101. Disponible en: http://dx.doi.org/10.5209/rev_CDMU.2016.v27.n1.52870.

BABINI, D. La comunicación científica en América Latina es abierta, colaborativa y no comercial. Desafíos para las revistas. Palabra clave, 2019, vol. 8, nº 2, e065. Disponible en: https://doi.org/10.24215/18539912e065.

BUNGE, M. La Ciencia, su Método y su Filosofía. 4ta edición. Buenos Aires, Argentina: Editorial Sudamericana, 2001.

CALVA CABRERA, K. y PUNÍN LARREA, M.I. Docentes ecuatorianos de comunicación: presencia y uso de las redes científicas académicas. Revista General de Información y Documentación, 2016, vol. 26, nº 2, p. 417-436.

CAMPOS, F. Las redes virtuales emergen como nuevas plataformas de gestión del conocimiento. Facultad de Ciencias de la Comunicación de la Universidad de Santiago, 2009. Disponible en: $<$ http://www.iiis.org/CDs2008/CD2009CSC/CCC2009/PapersPdf/D644IQ.pdf> [Consulta: 30 de junio de 2019]

CAMPOS FREIRE, F.; RIVERA ROGEL, D. y RODRÍGUEZ HIDALGO, C. La presencia e impacto de las universidades de los países andinos en las redes sociales digitales. Revista Latina de Comunicación Social, 2014, vol. 69, p. 571-592. Disponible en: https://doi.org/10.4185/RLCS-2014-1025.

CASTAÑO, C. (coordinador). Web 2.0. El uso de la web en la sociedad del conocimiento. Investigación e implicaciones educativas. Caracas, Venezuela: Universidad Metropolitana, 2009.

CÉSPEDES VILLEGA, A.; PERALTA GONZÁLEZ, M. y PAZ ENRIQUE, L. Indicadores alternativos de la actividad científica de la Universidad Central «Marta Abreu» de Las Villas en la Web 2.0. Cuadernos de Documentación Multimedia, 2017, vol. 28, $\mathrm{n}^{\circ}$ 2, p. 113-134. Disponible en: http://dx.doi.org/10.5209/CDMU.57070.

DORTA-CONTRERAS, A. Colaboración internacional y visibilidad de la producción científica por las métricas alternativas. Revista Habanera de Ciencias Médicas, 2016, vol. 15, nº 6, p. 859-863.

ENRÍQUEZ, S.; GARGIULON, S.; VERDECIA, E. y WENK, N. Circulación de textos científicos en sitios web académicos no institucionales. III Jornadas de TIC e innovación en el aula. Buenos Aires, Argentina: 2015. 
FERNÁNDEZ-MARCIAL, V. y GONZÁLEZ-SOLAR, L. Promoción de la investigación e identidad digital: el caso de la Universidade da Coruña. El profesional de la información, 2015, vol. 24, nº 5, p. 656-664. Disponible en: http://dx.doi.org/10.3145/epi.2015.sep.14.

FERNÁNDEZ, D. Revistas científicas e índices de impacto. A propósito de “Hacer saber”. Área Abierta, 2008 , vol. 20, p. 1-10.

GONZÁLEZ-DÍAZ, C.; IGLESIAS-GARCÍA, M. y CODINA, L. Presencia de las universidades españolas en las redes sociales digitales científicas: caso de los estudios de comunicación. El profesional de la información, 2015, vol. 24, n ${ }^{\circ}$ 5, p. 640-647. Disponible en: http://dx.doi.org/10.3145/epi.2015.sep.12.

GUÉDON, J. Plataformas (como Redalyc), revistas, libros y artículos digitales. ¿Cómo abrir el campo de cuestiones científicas sin quedar atrapado por una lógica comercial? Palabra Clave, 2019, vol. 8, n 2, e064. Disponible en: https://doi.org/10.24215/18539912e064.

HERNÁNDEZ, L. Redalyc aliado para la visibilidad. Revista de Ciencias Sociales, 2015, vol. XXI, nº 3, p. $289-291$.

IGLESIAS-GARCÍA, M.; GONZÁLEZ-DÍAZ, C. y CODINA, L. Estudio de perfiles de Research Gate y Academia.edu de universidades españolas: El caso de los departamentos de Comunicación Audiovisual y Publicidad. De los medios a los metamedios. Actas I Simposio de la Red Internacional de Investigación de Gestión de la Comunicación. Pontevedra, España, 2015.

KREIMER, P. ¿Dependientes o integrados? La ciencia latinoamericana y la nueva división internacional del trabajo. Nómadas, 2006, vol. 24, p. 199-212.

LAAKSO, M. et al. Research Output Availability on Academic Social Networks: Implications for Stakeholders in Academic Publishing. Electronic Markets, 2017, vol. 27, $\mathrm{n}^{\mathrm{o}} \quad 2$, p. 125-133. Disponible en: https://doi.org/10.1007/s12525-016-0242-1.

NOORDEN, R. Online collaboration: Scientists and the social network. Nature, 2014, vol. 512, p. 126-129. Disponible en: https://doi.org/10.1038/512126a.

ORDUÑA-MALEA, E.; MARTÍN-MARTÍN, A. y DELGADO-LÓPEZ-CÓZAR, E. ResearchGate como fuente de evaluación científica: desvelando sus aplicaciones bibliométricas. El profesional de la información, 2016a, vol. 25, $\mathrm{n}^{\circ}$ 2, p. 303-310. Disponible en: http://dx.doi.org/10.3145/epi.2016.mar.18.

ORDUÑA-MALEA, E.; MARTÍN-MARTÍN, A. y DELGADO LÓPEZ-CÓZAR, E. Métricas en perfiles académicos: ¿un nuevo juego adictivo para los investigadores? Revista Española de Salud Pública, 2016b, vol. 90, nº 22 , e1-e5.

QUINTAS, N. La emergencia de las redes sociales académicas: su impacto académico. Opción, 2016, vol. 32, nº 10, p. 517-528.

ROBINSON-GARCÍA, N.; REPISO, R. y TORRES-SALINAS, D. Perspectiva y retos de los profesionales de la evaluación científica y la bibliometría. El profesional de la información, 2018, vol. 27, $\mathrm{n}^{\circ}$ 3, p. $461-466$. Disponible en: https://doi.org/10.3145/epi.2018.may.01.

ROMERO-RODRÍGUEZ, L. Redes sociales científicas: ResearchGate. Comunicar: Escuela de Autores [en línea]. 2016. Disponible en: <https://comunicarautores.com/2016/12/08 /redes-sociales-cientificas-1-research-gate/> [Consulta: 30 de junio de 2019]

ROMERO-RODRÍGUEZ, L. Redes sociales científicas: Academia.edu. Comunicar: Escuela de Autores [en línea]. 2017. Disponible en: <https://comunicarautores.com/2017/06/26 /redes-sociales-cientificas-academia-edu/> [Consulta: 30 de junio de 2019]

SÁNCHEZ-JUÁREZ, I. Capítulo III: Publicar y difundir con éxito en las ciencias sociales. Tendencias en estudios de la información, comunicación e investigación. Editorial Alfagrama [en línea]. 2018. Disponible en: <https://www.researchgate.net/publication/331628550_Publicar_y_difundir_con_exito_en_las_ciencias_sociales> [Consulta: 30 de junio de 2019]

SUGIMOTO, C.; WORK, S.; LARIVIÈRE, V. y HAUSTEIN, S. Scholarly use of social media and altmetrics: a review of the literatura. Preprint submitted to JASIST [en línea]. 2016. Disponible en: $<$ https://arxiv.org/abs/1608.08112> [Consulta: 30 de junio de 2019]

TENNANT, J. et al. Ten Hot Topics around Scholarly Publishing. Publications, 2019, vol. 7, n $\mathrm{n}^{\mathrm{O}}$ 34, p. 1-24. Disponible en: <https://www.mdpi.com/2304-6775/7/2/34> [Consulta: 30 de junio de 2019]

VALENZUELA, C. En qué consiste AmeliCA, la alternativa de acceso abierto para América Latina. Tipográfica [en línea]. 2019. Disponible en: <https://tipografica.cl/en-que-consiste-amelica-la-alternativa-de-acceso-abierto-paraamerica-latina/> [Consulta: 30 de junio de 2019]

WALTMAN, L. y TRAAG, V.A. Use of the journal impact factor for assessing individual articles need not be wrong [en línea]. 2017. Disponible en: <https://arxiv.org/abs/1703.02334> [Consulta: 30 de junio de 2019] 